\title{
1 MITRE: predicting host status from microbiota time-series data
}

3 Elijah Bogart ${ }^{1,2}$, Richard Creswell ${ }^{1}$, Georg K. Gerber ${ }^{1, *}$

$5{ }^{1}$ Massachusetts Host-Microbiome Center, Department of Pathology, Brigham and

6 Women's Hospital, Harvard Medical School, 60 Fenwood Road, Boston, MA,

7 USA

8 2Present address: Kintai Therapeutics, Inc., 55 Cambridge Parkway Suite 800E,

$9 \quad$ Cambridge, MA 02142

$10 *$ Corresponding author, ggerber@bwh.harvard.edu

\section{Abstract}

13 Longitudinal studies are crucial for discovering casual relationships between the

14 microbiome and human disease. We present Microbiome Interpretable Temporal

15 Rule Engine (MITRE), the first machine learning method specifically designed for

16 predicting host status from microbiome time-series data. Our method maintains

17 interpretability by learning predictive rules over automatically inferred time-periods

18 and phylogenetically related microbes. We validate MITRE's performance on

19 semi-synthetic data, and five real datasets measuring microbiome composition

20 over time in infant and adult cohorts. Our results demonstrate that MITRE performs

21 on par or outperforms "black box" machine learning approaches, providing a 
22 powerful new tool enabling discovery of biologically interpretable relationships

23 between microbiome and human host.

25 Keywords: microbiome, time-series, longitudinal, machine learning

\section{Introduction}

28 The human microbiome is highly dynamic on multiple timescales, changing

29 dramatically during development of the gut in childhood, with diet, or due to

30 medical interventions [1]. Recently, a number of longitudinal studies have been

31 undertaken, seeking to link changes in the microbiota over time with medical

32 interventions such as delivery by Cesarean section [2], dietary changes [3], or

33 antibiotic treatment [4], or with disease outcomes in the host such as type 1

34 diabetes [5], dietary allergies [6], premature delivery [7, 8], necrotizing enterocolitis

$35 \quad[9,10]$ and infection $[11,12]$.

37 Deriving maximally useful information from these studies requires computational

38 methods that can simultaneously identify patterns of change in the microbiome and

39 link these patterns to the host's status (e.g. disease outcome, presence or absence

40 of an intervention, etc.) Moreover, such computational methods must contend with

41 numerous challenges inherent to microbiome time-series data, including 
42 measurement noise, sparse and irregular temporal sampling, and inter-subject

43 variability.

45 To overcome the challenges inherent in analyzing microbiome time-series data to

46 predict host status, we developed MITRE, a computational model that infers

47 human-interpretable predictive rules from high-throughput microbiome time-series

48 data, implemented in an open-source software package

49 (https://github.com/gerberlab/mitre/). MITRE falls into the general category of

50 Bayesian supervised machine learning classifiers: the algorithm uses a training

51 dataset of microbiota time-series and host statuses (supervised learning) to learn

52 a probability distribution (Bayesian inference) over a set of alternative models that

53 predict the status of a host from associated microbiome data (classification).

54 Bayesian approaches are powerful, because they provide principled estimates of

55 uncertainty throughout the model, which is an especially important feature in

56 biomedical applications where noisy inputs are the norm. We note that another

57 rule-based method, association rule mining (ARM), has recently been applied to

58 analyzing microbiome data in a different context (finding interaction patterns

59 among OTUs) [13]. Although ARM has some commonalities with Bayesian rule

60 learning approaches, ARM methods tend to employ user-based cut-offs and

61 heuristics, rather than principled probabilistic methods, as their primary function is

62 to mine large databases for putative interactions, rather than build predictive

63 models. Further, unlike Bayesian models, ARM methods do not incorporate prior

64 knowledge, as their focus is mining from large databases. 
67 In previous work, we presented the MDSINE [14] algorithm, which infers dynamical

68 systems models from microbiome time-series data in order to predict population

69 dynamics of the microbiome over time. Our present work, MITRE, addresses a

70 different question: can we predict the status of the host given microbiome time-

71 series data. From the machine learning perspective, MDSINE is an unsupervised

72 model, whereas MITRE is a supervised model. The key distinction is that MDSINE

73 models the microbiome, whereas MITRE does not, and instead models host

74 outcomes. Like other supervised models, MITRE focuses on finding only the

75 essential features (in this case, microbial clades and relevant time-windows) to

76 explain the outcome, rather than attempting to explain the microbiome data itself.

77 This architecture is ideal for highly heterogeneous datasets with many

78 "distractors," which are the reality for longitudinal studies of the human

79 microbiome. Supervised machine learning classifiers are employed in many

80 biomedical predictive modeling applications, including forecasting (predicting a

81 future outcome, such as onset of disease, based on past data) and diagnosis or

82 subtyping (predicting which category a subject belongs to based on all available 83 data).

85 MITRE's unique contributions are its modeling of the special properties of 86 microbiome time-series data (phylogenetic and temporal relationships), and its 
87 emphasis on producing human-interpretable predictors. This latter capability is in

88 contrast to various generic "black box" machine learning methods that have been

89 applied to analyzing static microbiome data, such as random forests [6, 15-17],

90 which may achieve high predictive accuracy but do not yield easily human-

91 interpretable models; interpretability is especially challenging for such models in

92 the context of time-series analyses, given repeated measurements and the fact

93 that relevant dynamics may occur at multiple time-scales. In the following sections,

94 we introduce the MITRE framework, then provide benchmarking results of MITRE

95 versus comparator methods on semi-synthetic data and five real microbiome time-

96 series datasets, and finally illustrate through examples MITRE's exploratory data

97 analysis capabilities and how these can help extract biological insights.

99 Results

100 Conceptual overview of the MITRE model and software

101 Figure 1 provides an overview of the MITRE framework. MITRE takes as input: (1)

102 tables of microbial abundances, typically operational taxonomic units (OTUs) from

103 16S rRNA amplicon sequencing or species mappings from metagenomic data,

104 measured over time for each host, (2) the status of each host (e.g., phenotype A

105 or phenotype B), (3) an optional set of static covariates for each host (e.g., gender),

106 and (4) placements of the microbes on a reference phylogenic tree [18]. MITRE

107 then automatically learns from the data predictive models that can be expressed

108 as a set of conditional statements, or human-readable rules, about time-localized 
109 patterns of change in the abundances of groups of phylogenetically related

110 organisms. Weighted sums of the truth values of the rules are used to predict the

111 status of each host. The MITRE software package also provides a graphical user

112 interface (GUI) for interactive visualization of the output, which summarizes the

113 predictive models learned from the data.

115 To be more precise, a MITRE model consists of a baseline probability of a default

116 host status plus a set of zero or more rules. Each rule is a conjunction of one or

117 more detectors-conditional statements about bacterial abundances in the form

118 "between times $t_{0}$ and $t_{1}$, the average abundance of bacterial group $j$ is

119 [above/below] threshold $\theta_{1}$ " or "between times $t_{0}$ and $t_{1}$, the slope of the abundance

120 of bacterial group $j$ is [above/below] threshold $\theta_{i}$-'together with a multiplicative

121 effect on the odds of the outcome of interest if all the detectors are satisfied.

123 As a simple example, a MITRE model predicting the odds of an infant developing

124 a disease in the first year of life might be:

125 - If, from month 2 to month 5 , the average relative abundance of bacterial 126 clade $\mathrm{A}$ is above $4.0 \%$, and from month 5 to month 8 , the relative abundance 127 of bacterial clade $B$ increases by at least $1.0 \%$ per month, the odds of 128 disease increase by a factor of 10 ;

129 - If, from month 3 to month 10, the average relative abundance of OTU $\mathrm{C}$ is less than $9.5 \%$, the odds of disease decrease by a factor of 2 ; 
131 - The baseline probability of disease is $22.0 \%$.

132 Figure 1B schematically illustrates the application of a rule set to hypothetical data.

133 To predict the probability that an individual will develop the disease, the effects of

134 each rule satisfied by that individual's microbiome data are combined with the

135 baseline disease probability.

137 A comprehensive pool of possible detectors is generated automatically at the

138 beginning of a MITRE analysis, including detectors that apply to average values

139 and rates of change of clades at all levels on the phylogenetic tree of observed

140 bacteria at as many time windows as the temporal resolution of the data will allow

141 (see Methods.) By combining detectors from this pool, rules in a MITRE model can

142 capture rich temporal patterns, but still remain human-interpretable because each

143 component rule is easy to understand.

145 MITRE is a nonlinear model, which has a number of advantages over a linear

146 model. Most obviously, nonlinear models can capture effects such as

147 thresholds/saturation or interactions between variables. A more subtle issue,

148 particularly relevant to microbiome ratio-based data, is that linear models introduce

149 mathematical/statistical difficulties when analyzing compositional or proportional

150 data. While special constraints are needed for linear models to overcome these

151 difficulties [19], nonlinear models do not suffer from the same limitations because

152 they can inherently learn nonlinear transformations of the data. It is particularly 
153 straightforward to understand the transformations produced by the MITRE

154 detectors described above: the learned thresholds effectively discretize ratio data

155 into distinct levels, a transformation that renders the data mathematically non-

156 compositional.

158 The MITRE framework is fully Bayesian, meaning it learns a distribution over

159 models, called the posterior probability distribution, which takes into account both

160 the input data as well as prior information provided. In the case of MITRE, this prior

161 information favors by default parsimonious explanations, i.e., short sets of simple

162 rules. Importantly, the default prior is designed to favor the empty rule set, or the

163 case in which the baseline odds only are used to predict host status, and the

164 microbiome data plays no role. This feature of the default prior is designed to guard

165 against over-fitting. Moreover, through the formalism of Bayes factors (see

166 Methods), MITRE provides a quantitative measure for the evidence favoring no

167 association between the microbiome data and host status or the alternative of any

168 rule sets that predict such a relationship; this feature allows the user to rigorously

169 evaluate whether sufficient signal is present in the microbiome data to predict host

170 status. Of note, this measure in effect incorporates multiple "hypotheses"

171 simultaneously (as the inference procedure explores the entire space of possible

172 rule sets at once.) Additional Bayes factors allow the user to assess the evidence

173 that each particular bacterial clade or OTU is associated with the host status, by

174 comparing the evidence for a model in which no detector in the rule set applies to 
175 the clade of interest to a model in which at least one detector does apply to the

176 clade.

178 The MITRE software approximately infers the posterior probability distribution

179 using a custom Markov Chain Monte Carlo algorithm, and reports a point estimate

180 of the single best rule set as well as a summary of the distribution, which the user

181 may investigate interactively with the provided GUI. To make predictions for new

182 data, either the point estimate or an ensemble of multiple rule sets, weighted

183 according to their posterior probabilities, may be used.

185 Benchmarking against standard machine learning methods: semi-synthetic data

186 We used semi-synthetic data to compare the cross-validated predictive 187 performance of MITRE to two popular standard machine learning methods, 188 random forests and L1-regularized logistic regression, which have been widely 189 used to analyze data from static microbiome studies. We tested the performance

190 of MITRE and the comparator methods by training each method on a subset of the

191 available data and then classifying the remaining data according to the model

192 learned. For each method, performance was evaluated using the F1-score

193 (harmonic mean of precision and recall) under crossvalidation, converting modeled

194 probabilities of outcomes to binary predictions by applying a threshold at 195 probability 0.5 . 
197 We simulated data from a real dataset using a parametric bootstrapping-type

198 procedure, in which models of microbiome dynamics were employed to interpolate

199 the real data and inject temporal perturbations into microbial clades to simulate a

200 "disease" host phenotype (see Methods for details). The real dataset [2] we

201 bootstrapped from tracked the gut microbiome composition from birth to two years

202 of age in a cohort of U.S. infants; we chose this dataset because it was among the

203 densest and most regularly sampled of available time-series datasets, and also

204 studied a relatively large number of subjects. To gain insight into the predictive

205 performance of the different methods, we simulated data with varying numbers of

206 subjects or time-points, and one or two temporal perturbations to microbial clades

207 to simulate subjects with a "disease." We assumed the perturbations occur over a

208 limited but unknown time-period during the study ( $20 \%$ of the study duration),

209 which represents a challenging but biologically relevant scenario for analysis. The

210 ranges of subjects and time-points simulated correspond approximately to those

211 in real studies, and thus provide insight into the performance of the algorithms on

212 realistically sized studies. Note that MITRE is a supervised machine learning

213 method, which does not directly model the covariates (microbiome data.) Thus,

214 our data simulation procedure is unrelated to the underlying MITRE model and not

215 expected to introduce bias in favor of our method.

217 To provide as reasonable a comparison as possible, we used the average 218 abundance of each OTU in a series of time windows as input to the comparator 219 algorithms. It is important to note that there are no prior methods available that 
220 were specifically designed for supervised learning from microbiome time-series

221 data. In fact, the comparator algorithms we implemented themselves represent an

222 advance over the state-of-the-art for many studies. To date, most studies have

223 employed an ad hoc strategy of manually identifying time windows of interest within

224 the experiment, and then testing for differential abundance of pre-specified groups

225 of taxa in each time window separately. Such an approach has significant

226 limitations. For example, effects occurring outside defined windows or across their

227 boundaries may not be detected; and, analyzing each time window/taxon pair

228 independently significantly reduces statistical power and precludes the discovery

229 of interactions across taxa or sequences of events across multiple time windows.

231 Our results on semi-synthetic data over a range of scenarios (Figure 2A-2D)

232 demonstrate superior cross-validated predictive performance of MITRE compared

233 to the other methods. Several interesting trends are also evident from these

234 results. First, the MITRE ensemble and point methods have similar performance,

235 except for in the setting of low numbers of subjects, which is likely simply a

236 stochastic effect since all the methods in that setting have poor performance. The

237 similar performance of the MITRE point and ensemble methods is very

238 encouraging from the interpretability perspective, since the point method yields a

239 single, human-interpretable rule set. Second as expected, all of the methods

240 improve in performance with increasing numbers of subjects, with eventual

241 plateauing of gains in performance at a level ultimately limited by noise in the data.

242 Third, there is also improvement in performance with increasing numbers of time- 
243 points, but this improvement is less impressive. This phenomenon can be partially

244 explained by our assumption in generating the semi-synthetic data that

245 perturbations corresponding to the "disease" phenotype occur over a limited time-

246 period during the study. Thus, sampling of more time-points outside the

247 perturbation period provides only limited additional information useful for

248 prediction. Fourth and finally, we also see generally worse performance of all

249 methods in the more complex setting of two perturbations in the "disease" cases,

250 particularly with limited numbers of subjects or time-points. Interestingly, Random

251 Forests outperforms L1-regularized logistic regression in the two-perturbation case

252 in the setting of low numbers of subjects or time-points, while the opposite is true

253 in the one-perturbation case, which may be due to Random Forest's capacity to

254 handle non-linearities. In any event, MITRE, which models non-linearities through

255 conjunctions in rules, consistently outperforms the other two methods in this setting

256 as well. Overall, our results demonstrate that MITRE, a method specifically tailored

257 for analyzing microbiome time-series data, outperforms generic machine learning

258 methods. Moreover, we provide a simulation and testing platform for users to

259 investigate questions relevant to particular microbiome time-series datasets in the 260 future.

262 Benchmarking against standard machine learning methods: real data

263 We next evaluated the performance of MITRE on real experimental datasets with

264 16S rRNA amplicon and whole-genome-shotgun metagenomic sequencing data,

265 from five representative published studies with sufficiently dense temporal 
266 sampling and numbers of subjects. Vatanen et al. [6] tracked gut microbiome 267 composition and life history data, including allergy diagnoses and serum IgE levels,

268 from birth to three years of age in cohorts of infants at high risk for autoimmune 269 disease in Finland, Estonia, and Russia. David et al. [3] tracked the gut microbiome 270 composition of healthy adults before, during and after a five-day period of 271 consuming exclusively plant-based or exclusively animal-based diets. Bokulich et 272 al. [2] tracked the gut microbiome composition from birth to two years of age in a 273 cohort of U.S. infants and examined the effects of mode of delivery, diet, and 274 antibiotic exposure. Kostic et al. [5] tracked the gut microbiome of Finnish and 275 Estonian infants at high risk for type 1 diabetes throughout the first years of life, 276 examining microbiome correlates of disease development. Finally, DiGiulio et al. 277 [7] tracked the composition of the vaginal microbiome in a cohort of pregnant 278 women, investigating an association with premature delivery.

280 Using the microbiome and outcome/class data from these five studies, we defined 281 a total of eleven representative microbiome-based prediction or classification tasks 282 (e.g., given the vaginal microbiome data of DiGiulio et al., predict which women in 283 the cohort experienced premature delivery; given the gut microbiome data of David 284 et al, determine which time series correspond to exclusively animal-based diets vs 285 exclusively plant-based diets; etc.) A full list of the tasks analyzed is given in 286 Supplementary Table 1. In five out of the eleven tasks, we found that at least one 287 of the methods performed well (F1-score $>0.7$, indicating reasonably high 288 precision and recall.) These tasks represent scenarios in which true biological 
289 signal may be present in the data, and thus serve as the most meaningful basis

290 for comparing performance of the different methods. Detailed results for all

291 methods on all tasks are given in Supplementary Table 1.

292

293 Both the MITRE point estimate and ensemble method achieved high accuracy on

294 all five of the relevant prediction or classification tasks (Figure 2E). In the case of

295 distinguishing infants fed formula-based diets from those predominantly breast-fed

296 (Bokulich et al.), predicting seroconversion to serum autoantibody positivity in

297 infants at high risk of T1D (Kostic et al.), and predicting premature delivery

298 (DiGiulio et al.), MITRE significantly outperformed the random forest and L1-

299 regularized logistic regression approaches; for Russian cohort membership

300 (Vatanen et al.) or plant-based diet (David et al.) prediction, MITRE performed on

301 par with the best comparator method (Random Forest). These results are

302 consistent with our semi-synthetic data simulations as well. Collectively our results

303 suggest that MITRE's phylogenetic aggregation approach and robust use of the

304 temporal structure of the data provide significant advantages for classification, and

305 the increased interpretability of the MITRE point estimate comes at little if any cost

306 in predictive accuracy.

308 Model interpretability and exploratory analysis capabilities

309 We illustrate here an example demonstrating MITRE's ability to achieve high 310 accuracy while maintaining interpretability. The best (point estimate) rule set 
311 learned by MITRE to distinguish between predominantly formula-fed and

312 predominantly breast-fed infants in the study of Bokulich et al. [2] is "If, between

313 the $1^{\text {st }}$ day of life and the $156^{\text {th }}$ day of life, the average abundance of clade 13241

314 increases faster than $0.03 \%$ per day, the probability that the subject was

315 predominantly formula-fed is $79 \%$; otherwise that probability is $5.4 \%$." Though this

316 MITRE rule set is simple enough to express in a single sentence, it outperforms

317 Random Forest models that aggregate the predictions of over a thousand decision

318 trees (Figure 2E). Moreover, this MITRE rule set lends itself readily to biological

319 interpretation. Clade 13241 is a broad group of Firmicutes, including OTUs in the

320 dataset mapping to Ruminococcus gnavus, Roseburia hominis, and several

321 Clostridium and Blautia species. These species are generally viewed as more

322 representative of adult or at least more mature microbiomes, being strict

323 anaerobes with specialized carbon source utilization requirements and capabilities

324 (e.g., [20]), suggesting that the formula diet may shift infants toward more adult-

325 like gut microbiota. Of note, the expressiveness and interpretability of the MITRE

326 rule set format is retained even in cases of nonlinear interactions across multiple

327 clades and time windows; see the Supplementary Note for an example.

329 In addition to providing the point estimate, which can serve as a powerful predictive 330 model as described, MITRE also allows the user to explore the distribution of

331 probable rules learned by the framework. Such explorations can be useful for 332 further interpreting rule sets and generating biological hypotheses. Figure 3 333 illustrates MITRE's capabilities for interactive visualization of the distribution of 
334 learned rules. Heat maps as shown in Figure 3A and 3D allow the user to examine

335 the time windows and regions of the phylogenetic tree where the temporal changes

336 in the microbiota are most strongly associated with the outcome of interest. In

337 Figure $3 \mathrm{~A}-\mathrm{C}$, MITRE has been used to learn rules that distinguish the microbial 338 dynamics observed when the subjects in the study of David et al. [3] were fed an

339 exclusively plant-based diet for five days versus the dynamics observed when

340 subjects were fed an exclusively animal-derived diet. The user has clicked on two

341 areas on the heat map, revealing rules that apply to different time windows and

342 different groups of OTUs in the order Clostridiales. The first rule set pertains to a

343 clade containing Roseburia species, which are butyrate producers, whereas the

344 second rule set pertains to a clade containing Dorea species, which produce other

345 short-chain fatty acids including acetate and formate, but not butyrate. Thus, this

346 capability to explore the distribution of rule sets allows the user to find evidence

347 that the animal-based diet promotes two groups of phylogenetically distinct, and

348 likely functionally distinct, groups of microbes.

350 As another example, Figures 3D and 3E demonstrate exploration of the posterior

351 distribution of rule sets identifying temporal patterns that distinguish the microbiota

352 of predominantly formula-fed and predominantly breast-fed infants in the study of

353 Bokulich et al. [2], for which the point estimate described above performed well.

354 The heat map of Figure 3D shows that the high-posterior-probability rule sets are

355 strongly focused on a single group of OTUs in the first 156 days of life. Figure 3E

356 presents a particular detector, included in many such rule sets, that discriminates 
357 effectively between diet types in the training data (which is the detector used by

358 MITRE to form the point estimate rule discussed above). Thus, in this case, the

359 user finds evidence that the posterior distribution is essentially unimodal, with the

360 point estimate alone characterizing temporal differences between the formula and

361 breast-milk fed infants well.

\section{Discussion}

364 MITRE offers a number of advantages over generic statistical or machine learning 365 methods. Incorporation of phylogenetic information readily allows for biological 366 interpretation of results, as discussed, whereas standard classification methods 367 that evaluate each taxon independently clearly do not have this advantage. MITRE 368 automatically learns time windows that are relevant to predicting host status, as 369 opposed to generic approaches that require the user to manually specify periods 370 of interest, which are generally unknown a priori. We have also highlighted the

371 utility of MITRE's human-readable rules. These rules can capture rich temporal 372 patterns and nonlinear relationships among microbes, but remain interpretable, as 373 they are composed of simple and understandable detectors. Indeed, as we have 374 shown, MITRE rule sets are not only easy to understand, but a single MITRE rule 375 can outperform black box machine learning methods that make predictions based 376 on collections of hundreds to thousands of components, which are difficult to 377 understand even individually. 
379 Another important feature of the MITRE framework is that it is fully Bayesian.

380 Bayesian models are increasingly being adopted in a variety of fields, in particular

381 for biomedical applications (e.g., [21]), because they provide a unified framework

382 that handles a number of key modeling and inferential issues, including

383 incorporation of prior knowledge, accurate estimation of confidence in predictions,

384 and principled comparisons of multiple models. Bayesian methods for model

385 comparison have recently been highlighted as powerful alternatives [22] to

386 traditional $p$-value based hypothesis testing [23], because Bayesian approaches

387 allow direct comparison of multiple relevant alternative models, rather than just the

388 ability to reject a null model that is often of little interest in itself. In particular, MITRE

389 facilitates principled model comparisons by calculating Bayes factors [24], which

390 quantify the strength of the evidence provided by the data for each of a set of

391 competing models. We have also described how exploration of the posterior

392 distribution of rule sets using the provided GUI in the MITRE software package

393 allows the user to evaluate the possibility of multiple informative rule sets and

394 formulate biological hypotheses about the data set.

396 Although MITRE as currently implemented is primarily designed to use taxonomic 397 abundance profiles derived from 16S rRNA amplicon sequencing or WGS 398 metagenomics data as input-currently the most common data types in longitudinal 399 microbiome studies-the model and software can readily incorporate other time400 series of additional data types, e.g., host physiological measurements, 401 metabolomics, or metagenomics-derived functional profiles, as such data become 
402 more widely available. Other model features that could be readily added in the

403 future include time-varying, continuous, and multiple host outcomes, e.g.,

404 multivariate host read-outs such as blood pressure, blood glucose, and body 405 weight.

406

407 Conclusion

408 We have demonstrated that our software package MITRE overcomes the unique

409 challenges of predicting host outcomes from microbiome time-series data, while

410 drawing on a well-established tradition of rule-based techniques in machine

411 learning and artificial intelligence [25-27], and can perform as well as or better than

412 "black box" machine learning methods while maintaining interpretability. This latter

413 feature is critical as microbiome analyses move into clinical applications, in which

414 patients and physicians necessarily place a premium on transparency and

415 interpretability of results. We have provided an open source and user-friendly

416 implementation of our method, which we expect will greatly aid investigators

417 analyzing longitudinal host-microbiome studies and ultimately provide novel

418 insights into the complex interplay between microbiome dynamics and host health

419 and disease.

420

421 Methods

422 


\section{Operation of the software and input data requirements}

424 The MITRE software is implemented in Python 2.7.3. MITRE and its dependencies

425 are available through the Python Package Index, pypi.python.org, facilitating

426 installation across multiple platforms. The software is run from the command-line,

427 with parameters and other inputs specified using a straightforward configuration

428 file format.

430 Each MITRE run requires four input files (for the standard case of $16 \mathrm{~S}$ rRNA

431 amplicon data): a table of OTU abundances in each sample, a table specifying the

432 subject and timepoint associated with each sample, a table specifying the outcome

433 (and optionally other data) associated with each subject, and phylogenetic

434 placements of the OTUs on a reference tree. The user provides the three tables in

435 comma-separated-value format, and phylogenetic placements in the .jplace format

436 produced by pplacer. Alternative input data types, including taxonomic abundance

437 profiles generated from WGS metagenomic data with Metaphlan, are described in

438 the MITRE manual online.

440 The output of the software, described in detail below, includes textual summaries

441 of a single best set of rules (the point estimate) and the distribution of probable

442 alternative rule sets, as well as an HTML file providing a graphical interface for

443 interactive visualization of the results. 


\section{MITRE model details}

446 Mathematical basis of the MITRE model

447 MITRE can be expressed as a hierarchical generative model that generates sets

448 of rules of the form described above. The generative process, starting at the top of

449 the hierarchy, is as follows:

450 - Sample a length $K$ for the rule set

451 - For each rule $k \in\{1, \ldots, K\}$, sample:

○ weight $\beta_{k}$

- A rule length $L_{k}$ (number of detectors in the rule)

○ The detectors $d \in\left\{1, \ldots, L_{k}\right\}$, drawing from a pre-defined pool of detectors (see below) according to a probability distribution which is parameterized by the time window and bacterial group (phylogenetic subtrees) to which each detector applies.

458 The rules are then weighted in a Bayesian logistic regression model to predict host

459 status. To be precise, assume we have observations $x_{i j t}$ for $i=1,2, \ldots, N_{\text {subjects }}$ of

460 relative abundances of bacterial OTUs $j=1,2, \ldots$, NOTUs sampled at time-points

$461 t=1,2, \ldots, N_{\text {samples }, i}$, as well as a binary status variable $y_{i}$ for each host. The MITRE

462 probability model can then be expressed as:

$$
y_{i} \sim \operatorname{Bernoulli}\left(p_{i}\right)
$$

464

$$
p_{i}=\frac{1}{1+e^{-\psi_{i}}}
$$

$$
\psi=\beta_{0}+A(R, x) \vec{\beta}
$$




$$
\beta \mid R \sim \operatorname{Normal}\left(0, \sigma_{b}^{2} I\right)
$$

$$
\beta_{0} \sim \operatorname{Normal}\left(0, \sigma_{b}^{2}\right)
$$

$$
R \sim \pi(R, x)
$$

469 Here, $R$ is a set of rules, and $A(R, x)$ is the matrix whose entry $A_{i k}$ is 1 if the data

470 from subject $i$ satisfies the conditions of all detectors in the $k$-th rule in the set, and

471 zero otherwise. Conditional on $R$, this is a standard Bayesian logistic regression

472 model, whose covariates are the truth values of the rules in $R$ (along with an offset

473 term modeling baseline odds). MITRE also allows the optional inclusion of static

474 non-microbiome covariates; see the Supplemental Note for complete details.

476 The prior probability distribution over rule sets, $\pi(R, x)$, is a mixture over the

477 probability $\Theta_{\text {empty }}$ for an empty rule set $R_{0}$, versus a truncated negative binomial 478 distribution for the length of a non-empty rule set. For a non-empty rule set, the 479 prior further models the distribution over detectors that comprise each rule, taking 480 into account the length of the time window for the detector and an associated 481 position on the phylogenetic tree. Hyperparameters for these priors, as well as 482 priors on other variables, complete the model. A full specification of priors in 483 MITRE, and a discussion of sensitivity to the choice of hyperparameters, are given 484 in the Supplementary Note. 
487 MITRE generates a comprehensive pool of detectors from the supplied data and 488 user-specified parameters $t_{\min }, t_{\max }, N_{\mathrm{w}}$, and $N_{\theta}$, as follows:

490 1. Divide the duration of the experiment/observations into $N_{w}$ equal basic time 491 windows, and enumerate all combinations of 1 or more consecutive basic 492 time windows that are longer than $t_{\min }$ and shorter than $t_{\max }$ and during which 493 at least one sample was collected for every subject.

2. Within each such time window $\left(t_{0}, t_{1}\right)$, for each bacterial group (phylogenetic subtree) $j$, calculate the average abundance of the group in each subject $i$, and sort those values, obtaining $v_{1} \leq v_{2} \leq \ldots \leq v_{\text {Nsubjects. If }} N_{\text {subjects }}<N_{\theta}+1$, for $I=1,2, \ldots, N_{\text {subjects }}-1$, let $\theta_{l}=\left(v_{l}+v_{l+1}\right) / 2$ and add the detectors "between $t_{0}$ and $t_{1}$, the average abundance of group $j$ is above $\theta_{1}$ and "between $t_{0}$ and $t_{1}$, the average abundance of group $j$ is below $\theta{ }^{\prime \prime}$ to the population. If instead $N_{\text {subjects }}>N_{\theta}$, hierarchically cluster the values $v_{0}, \ldots, v_{N \text { subjects }}$ into $N$ ${ }_{\theta}$ groups, let $\theta_{I}$ be the midpoint between cluster $I$ and cluster $I+1$, for $I=1,2, \ldots, N_{\theta}-1$, and add the detectors corresponding to those thresholds $504 \quad$ instead. 
508

509

510

511

512

513

514

515 The runtime of the inference algorithm (described below) depends approximately

516 linearly on the size of the detector pool; thus the choice of parameters $t_{\min }, t_{\max }, N_{\mathrm{w}}$,

517 and $N_{\theta}$ controls a tradeoff between high resolution (temporally, and in the space

518 of threshold values for OTU abundance/slope) and performance. It is

519 recommended to choose $N_{w}$ as large as possible while ensuring that most basic

520 time windows include at least one observation from every subject, and to set $N_{\theta}=$

52140 ; $t_{\min }$ should generally be set to 0 and $t_{\max }$ to either the duration of the study or

522 (to enforce temporal localization of the rules in cases where, e.g., dramatic

523 increases in abundance at a well-defined time also lead to notable increases in

524 average abundance over the period of the entire study) half that duration, but may

525 be adjusted if dynamics on a particular time scale are of a priori interest.

\section{Model inference}

528 We perform approximate Bayesian inference, to learn the posterior distribution 529 over model parameters including rule sets $R$ and regression coefficients $\beta$. MITRE 
530 employs a custom Markov Chain Monte Carlo (MCMC) algorithm, which alternates

531 efficient updates of the regression coefficients using a Polya-Gamma auxiliary

532 variable scheme [28], Metropolis-Hastings update steps that propose changes to

533 the rule set $R$, and updates to the hyperparameters governing the prior distribution

534 over rule sets. The MCMC algorithm is described in detail in the Supplementary

535 Note. Briefly, four types of updates to $R$ are considered:

1. A randomly-chosen detector in $R$ may be replaced by another detector from the pool

2. A randomly-chosen detector in $R$ may be removed from $R$ (if it is the only detector in a rule, the rule is removed as well)

3. A detector from the pool may be added to $R$, either to an existing rule chosen at random, or to form a new rule of length 1

4. A detector may be moved from one rule in $R$ to another

545 For the analyses presented here, 50,000 samples were drawn from the posterior

546 distribution (except for analyses of data from Vatanen et al. [6], where 25,000

547 samples were used, and Kostic et al. [5], where 100,000 samples were used.)

548 Mixing of the MCMC sampler was assessed using the diagnostics described in the

549 Supplementary Note; we recommend users employ these diagnostics to determine

550 the appropriate number of samples needed for their particular studies. 
552 Run time depends on the size and complexity of the data set; using a single Intel

553 Xeon CPU (E5-2697 v3 2.60GHz), sampling took 45 minutes for the data of

554 DiGiulio et al. [7], 23 hours for the data of David et al. [3], 30 hours for the data of

555 Bokulich et al. [2], and 64.5 hours for the data of Vatanen et al. [6]. Cross-validation

556 was performed in parallel (1 fold/core) requiring similar total elapsed time for each

557 study.

558

559 Data simulation

560 We simulated from the Bokulich et al. [2] dataset using a parametric bootstrapping-

561 style procedure. Simulated subjects were sampled with replacement from the set

562 of control subjects in the real data. Equal numbers of simulated cases and control

563 subjects were generated for each scenario. In order to have sufficient real data to

564 bootstrap to evaluate the range of scenarios of interest, we excluded subjects with

565 fewer than 13 time-points sampled, who had no samples before 10 days, and who

566 were studied for less than 600 days; this yielded 20 subjects for bootstrapping. We

567 then truncated data to an interval between 10 and 600 days, since this contained

568 the densest sampling across subjects.

570 Perturbations of duration 120 days, in time windows randomly occurring

571 throughout the experiment, were introduced into randomly selected clade(s) to

572 simulate cases, with the magnitude of the perturbation distributed among clade

573 members according to the relative abundance of members in the original data. The 
574 duration of the perturbation(s) was chosen to be approximately of the order of that

575 seen with the MITRE point rule on the real data set. Perturbations were introduced

576 randomly into clades with the following characteristics: minimum average relative

577 abundance of $0.1 \%$, maximum average relative abundance of $20 \%$, and a

578 maximum of 30 OTUs in the clade. These parameters were chosen so as to

579 provide a meaningful relative disturbance to other clades, but not to drastically

580 disrupt the entire microbiome (which would present less of a challenge to the

581 prediction algorithms.) The magnitude of perturbation(s) were sampled for each

582 subject from log-normal distributions, with mean and variance of the order of that

583 seen with the MITRE point rule on the real data set (control log mean $=-6$, control

$584 s t d=1.5$, case log mean $=-3$, case $\log s t d=1.5$ ). When two perturbations were

585 applied, each control subject received only one perturbation, whereas case

586 subjects received both perturbations.

588 Note that MITRE is a supervised learning (conditional) method, meaning that the

589 microbiome data itself is not modeled; to simulate time-points not present in the

590 original data set, we therefore must introduce a model of microbiome dynamics.

591 We model the underlying microbiome data as arising from latent time-dependent

592 stochastic processes (Gaussian random walks):

$$
x_{o s}(t) \sim \operatorname{Normal}_{+}\left(x_{o s}(t-1), \Delta t \tau^{2}\right)
$$

594 Here, $x_{o s}(t)$ is the latent trajectory for OTU $o$ in subject $s$ at time $t$, and $\tau^{2}$ is the 595 process variance parameter, which is empirically estimated from the real data as 
596 approximately the 75-percentile variance. We assume a Bayesian model and infer

597 the posterior latent trajectories using a 1-step ahead MCMC algorithm similar to

598 our previously described method [29], except in this case trajectories are assumed

599 to be independent of one another.

600

601 The observed data $c_{s}(t)$, consisting of sequencing counts, is assumed to arise 602 through a two-stage error model:

603

604

$$
y_{o S}(t) \sim \mathrm{Normal}_{+}\left(x_{o s}(t), \theta x_{o s}(t)\right)
$$

$$
m_{s}(t)=\sum_{o} y_{o s}(t)
$$

605

$$
C_{s}(t) \sim \operatorname{DMD}\left(y_{s}(t) / m_{s}(t), \alpha, N\right)
$$

606 Here, DMD denotes the Dirichlet-Multinomial distribution with concentration

607 parameter $\alpha$ and number of simulated sequencing reads per sample $N$; we use

608 parameters estimated from data $(a=286 ; N=50,000)$ as previously described 609 [14].

610

611 The model thus provides temporal coherence through the Gaussian random walk

612 latent trajectory, while modeling compositionality and over-inflation through the

613 two-stage error model. Posterior samples from the model capture temporal trends

614 seen in the real data (e.g., periods of time in which a particular OTU are 
615 increasing), but with randomness introduced so that subjects sampled with

616 replacement look sufficiently different.

617

618 For each scenario simulated (e.g., a particular number of time-points or subjects),

61910 independent simulations were performed. To investigate the effects of varying

620 the number of subjects, we fixed the simulation at an intermediate number of time-

621 points (18), and simulated different numbers of subjects. Similarly, to investigate

622 the effects of varying the number of time-points, we fixed the simulation at an

623 intermediate number of subjects (32), and simulated different numbers of time-

624 points. To facilitate comparisons, we sampled evenly spaced time-points in all

625 cases. The simulated data were then provided to MITRE and the other methods in

626 the same input format as for real data, as described below. Complete Python code

627 to reproduce the simulations is available in the MITRE online repository.

\section{Output}

630 MITRE generates several summaries from the posterior samples obtained from

631 the MCMC inference procedure described above. The point estimate is a single

632 rule set $R^{*}$ (with coefficients $\beta^{*}$ ) that summarizes the mode of the posterior

633 distribution. If the posterior probability that $R$ is empty is greater than 0.5 , the point

634 estimate is the empty rule set. Otherwise, to obtain a representative non-empty

635 summary list, we determine the posterior mode $d^{*}$ of the total number of detectors

636 in $R$, and take $R^{*}$ to be the rule set with the highest posterior probability among all 
637 sampled rule sets that contain $d^{*}$ total detectors. The point estimate coefficients $\beta^{*}$

638 are the highest posterior probability sampled coefficients associated with $R^{*}$.

640 To provide an overview of the possible alternative rule sets learned by the model, 641 rule sets in the posterior samples are clustered and a summary of the highest642 probability clusters is produced. The clustering process first forms clusters of

643 detectors that apply to the average value or slope of the same variable in highly 644 overlapping time windows (ignoring threshold values), then clusters together rules

645 whose component detectors belong to the same clusters, and finally groups rule 646 sets whose rules belong to the same clusters (see the Supplementary Note for a 647 full description.) For each cluster, a representative rule set and the estimated 648 posterior probability that $R^{*}$ belongs to the cluster are reported.

650 Calculation of the Bayes factor for the empty rule set $R_{0}$ (vs any non-empty $R$ ) and 651 two additional types of Bayes factors, indicating the relevance of phylogenetic 652 subtrees or time-windows, is described in the Supplementary Note.

654 Finally, MITRE generates an interactive graphical visualization of the posterior 655 distribution of rule sets. A heatmap of the Bayes factors for leaf variable/basic time 656 window combinations is rendered alongside the bacterial phylogeny (as in figure $6573 \mathrm{~A}, 3 \mathrm{D})$; clicking on any cell allows the visualization of the detectors associated 658 with the cell with the largest Bayes factors (as in figure 3B-C and 3E.) 
661 MITRE offers a number of user-configurable options for preprocessing and filtering 662 microbiome time-series data. The following procedure is recommended, and was 663 used for the results presented here (except as noted below):

665 1. To remove potentially spurious rare OTUs, discard all OTUs with less than $666 \quad N_{\text {counts, OTu observed across all samples (typically }} N_{\text {counts, OTU }}=10$.)

6672 2. To exclude samples where coverage is so low that abundance estimates 668 for uncommon OTUs may be unreliable, discard all samples with less than

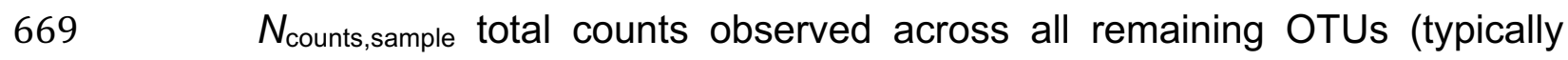
$N_{\text {counts,sample }}=5000$ for HiSeq/MiSeq data.)

3. If desired, to analyze only a particular time period (because, e.g., samples are not available outside that period for the majority of subjects) discard all samples before time $t_{i}$ and after time $t_{f}$ (by default, $t_{i}$ is the time of the earliest available sample, and $t_{f}$ the time of the latest sample.)

4. To exclude subjects for whom microbiome dynamics cannot be resolved at the desired temporal resolution throughout the entire study, discard subjects with too few, or too sparse, observation points, by dividing the duration of the study into $N_{\text {w, filter }}$ equal pieces and keeping only subjects with at least $N_{s}$ samples in any $N_{c}$ consecutive such pieces. Default values are $N_{\text {w,fiter }}=10, N_{s}=2$, and $N_{c}=1$; note that for data with very inconsistent 
681

682

683

684

685 After these steps, counts data are converted to relative abundance data for each

686 sample, and, for each node in the phylogenetic tree, a relative abundance estimate

687 is obtained by summing the relative abundances of its children. The following

688 filtering steps are then applied to all taxa (both OTUs and higher nodes in the tree): sampling, these parameters must be chosen judiciously to maximize the number of subjects included while allowing an acceptable level of temporal resolution.
690

700

701 
703 Note that, when a large number of taxa are considered, the detector pool becomes

704 large and the computational cost of the inference algorithm grows; if necessary, it

705 is recommended to increase the stringency of steps 5 and 6 to keep the total

706 number of taxa below 500.

708 Bioinformatics and preprocessing for analyzed datasets

709 For each 16S-based dataset to which MITRE was applied, the original 16S rRNA

710 amplicon sequencing data was reprocessed to obtain tables of OTU abundances

711 and phylogenetic placements for each OTU on a reference tree, using as

712 consistent an analysis process as possible given differences in sequencing

713 methodology and the nature of the available data. Where possible, DADA2 1.1.5

714 [30] was used to trim, quality filter, merge, and remove chimeras from the reads,

715 assign them to inferred true sequences, and classify the inferred sequences

716 taxonomically (such classification is not necessary for MITRE application, but is

717 helpful for interpretation of the results.) Inferred sequences were then placed on

718 a reference tree generated from full-length or near full-length (>1200 nt) 16S rDNA

719 sequences of type strains from the Ribosomal Database Project [31] using pplacer

720 [18]. When quality scores for sequences were not available and DADA2 could not

721 be used, sequences were instead processed using mothur 1.35.1 [32, 33] for

722 denoising, quality filtering, alignment against the ARB Silva 16 S gene sequence

723 reference database, clustering into OTUs at 97\% identity, and taxonomic

724 classification. For the WGS metagenomics data of Kostic et al [5], published

725 taxonomic abundance tables were used directly as input data to MITRE, exploiting 
726 MITRE's built-in support for parsing Metaphlan result tables, described in the

727 MITRE manual. Full details regarding pre-processing of all datasets are described

728 in the Supplemental Note.

730 After reanalyzing sequencing data for each study and excluding subjects with 731 infrequent sampling (see description of filtering methods above) we applied MITRE 732 and the comparator methods to classify subjects according to relevant categories:

733 membership in the Russian cohort $(n=30)$, elevated IgE levels $(n=28)$, diagnosis

734 with any allergy $(n=49)$, any dietary allergy $(n=42)$, with dairy allergy $(n=32)$, or with

735 egg allergy $(n=25)$ in the data of Vatanen et al. $(n=113$ total for nationality; $n=109$

736 total for all other outcomes); formula-dominant diet $(n=11)$ or Cesarean delivery

$737(n=13)$ in the data of Bokulich et al. $(n=35$ total); seroconversion $(n=11)$ in the data

738 of Kostic et al. ( $n=19$ total); premature delivery $(n=6)$ in the data of DiGiulio et al.

739 ( $n=37$ total); and plant-based diet $(n=10)$ in the data of David et al. $(n=20$ total.)

741 Comparison methods

742 To compare MITRE's predictive performance to alternative methods, each OTU's

743 abundance data for each subject was averaged across all observations within each

744 of a set of time windows, defined by dividing the experiment into $N_{\mathrm{w}, \text { comparison }}$ equal

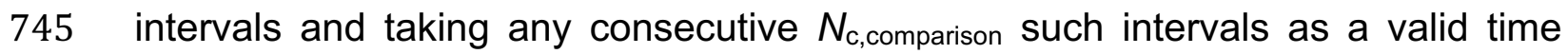

746 window. Parameters were chosen to maximize temporal resolution while ensuring

747 that each time window still contained at least one observation for each subject. 
748 Note that the same subjects were used (i.e., those not excluded by the

749 preprocessing settings described above) for both MITRE and the comparison

750 methods. For David et al. [3], $N_{\mathrm{w}, \text { comparison }}=10$ and $N_{\mathrm{c}, \text { comparison }}=3$; for Vatanen et al.

751 [6], $\quad N_{\mathrm{w}, \text { comparison }}=9$ and $N_{\mathrm{c}, \text { comparison }}=4$; for DiGiulio et al. [7],

$752 N_{\mathrm{w}, \text { comparison }}=N_{\mathrm{c}, \text { comparison }}=1$; for Bokulich et al. [2], $N_{\mathrm{w}, \text { comparison }}=12$ and $N_{\mathrm{c}, \text { comparison }}=2$;

753 for Kostic et al. [5], $N_{\mathrm{w}, \text { comparison }}=5$ and $N_{\mathrm{c}, \text { comparison }}=2$.

754

755 These averaged abundances were then used to train Random Forest or logistic

756 regression classifiers using the Python package scikit-learn [34]. Random Forest

757 classifiers included 1024 trees (as larger numbers of trees were not found to

758 improve classifier performance.) For logistic regression with $L 1$ regularization, the

759 regularization strength parameter was chosen using 10-fold crossvalidation from

760 among a grid of logarithmically spaced options spanning the range $10^{-4}$ to $10^{4}$.

761

762 Declarations

763

764 Acknowledgements: We thank Daniel DiGiulio for assistance with the data from

765 reference [7], and Travis Gibson for helpful comments on the manuscript.

766 Funding: This work was supported by the Brigham and Women's Hospital

767 Precision Medicine Initiative and NIH 5R01GM130777 to GKG. EB received 768 support from NIH grant T32HL007627. 
769 Availability of data and materials:

770 The MITRE software package is available at: https://github.com/gerberlab/mitre/ .

771 All additional input files needed to reproduce the results presented here, and

772 detailed output from all MITRE simulations discussed, are available at

773 https://github.com/gerberlab/mitre paper results.

774

775 Datasets analyzed during the current study are available: data from Bokulich et

776 al. [2], from ENA (https://www.ebi.ac.uk/ena/data/view/PRJEB14529) and QIITA

777 (https://qiita.ucsd.edu, study 10249); data from David et al. [3], from MG-RAST

778 (http://metagenomics.anl.gov/linkin.cgi?project=mgp6248); data from Kostic et al.

779 [5], via the DIABIMMUNE project web site

780 (https://pubs.broadinstitute.org/diabimmune/t1d-cohort); data from Vatanen et al.

781 [6], via the DIABIMMUNE project web site

782 (https://pubs.broadinstitute.org/diabimmune/three-country-cohort); data from

783 DiGiulio et al. [7], on request from those authors.

784 Authors' contributions: EB and GKG conceived the method, developed the theory,

785 and wrote the manuscript. EB wrote the software, curated the relevant data, and

786 formally analyzed and validated the method and software. RC contributed to the

787 software and analysis and validation of the method.

788 Ethics approval and consent to participate: Not applicable

789 Consent for publication: Not applicable 
790 Competing interests: GKG is a Strategic Advisory Board Member of Kaleido

791 Biosciences and has a sponsored research agreement with the company, and is

792 a Scientific Advisory Board Member, co-founder, and shareholder of ConsortiaTX.

793 No funding for the present work was provided by either company.

794

$795 \quad$ References

796

797 1. Gerber, G.K., The dynamic microbiome. FEBS Lett, 2014. 588(22): p. 4131-9.

798 2. Bokulich, N.A., et al., Antibiotics, birth mode, and diet shape microbiome

799 maturation during early life. Sci Transl Med, 2016. 8(343): p. 343ra82.

8003 3. David, L.A., et al., Diet rapidly and reproducibly alters the human gut

801 microbiome. Nature, 2014. 505(7484): p. 559-63.

802 4. Yassour, M., et al., Natural history of the infant gut microbiome and impact of

803 antibiotic treatment on bacterial strain diversity and stability. Sci Transl Med,

$804 \quad 2016.8(343):$ p. 343ra81.

805 5. Kostic, A.D., et al., The dynamics of the human infant gut microbiome in

806 development and in progression toward type 1 diabetes. Cell Host Microbe,

807

808

809

810

811

812

813

814

815

816

817

818

819

820

821

822

823

824

825 2015. 17(2): p. 260-73.

6. Vatanen, T., et al., Variation in Microbiome LPS Immunogenicity Contributes to Autoimmunity in Humans. Cell, 2016. 165(4): p. 842-53.

7. DiGiulio, D.B., et al., Temporal and spatial variation of the human microbiota

during pregnancy. Proc Natl Acad Sci U S A, 2015. 112(35): p. 11060-5.

8. $\quad$ Romero, R., et al., The vaginal microbiota of pregnant women who subsequently have spontaneous preterm labor and delivery and those with a normal delivery at term. Microbiome, 2014. 2: p. 18.

9. Raveh-Sadka, T., et al., Gut bacteria are rarely shared by co-hospitalized premature infants, regardless of necrotizing enterocolitis development. Elife, 2015. 4.

10. Zhou, Y., et al., Longitudinal analysis of the premature infant intestinal microbiome prior to necrotizing enterocolitis: a case-control study. PLoS One, 2015. 10(3): p. e0118632.

11. Pop, M., et al., Individual-specific changes in the human gut microbiota after challenge with enterotoxigenic Escherichia coli and subsequent ciprofloxacin treatment. BMC Genomics, 2016. 17: p. 440.

12. van Rensburg, J.J., et al., The Human Skin Microbiome Associates with the Outcome of and Is Influenced by Bacterial Infection. MBio, 2015. 6(5): p.

826 e01315-15. 
827 13. Tandon, D., M.M. Haque, and S.S. Mande, Inferring Intra-Community Microbial

828

829

830

831

832

833

834

835

836

837

838

839

840

841

842

843

844

845

846

847

848

849

850

851

852

853

854

855

856

857

858

859

860

861

862

863

864

865

866

867

868

869

870

871

872
Interaction Patterns from Metagenomic Datasets Using Associative Rule Mining Techniques. PLoS One, 2016. 11(4): p. e0154493.

14. Bucci, V., et al., MDSINE: Microbial Dynamical Systems INference Engine for microbiome time-series analyses. Genome Biol, 2016. 17(1): p. 121.

15. Subramanian, S., et al., Persistent gut microbiota immaturity in malnourished Bangladeshi children. Nature, 2014. 510(7505): p. 417-21.

16. Sze, M.A. and P.D. Schloss, Looking for a Signal in the Noise: Revisiting Obesity and the Microbiome. MBio, 2016. 7(4).

17. Teng, F., et al., Prediction of Early Childhood Caries via Spatial-Temporal Variations of Oral Microbiota. Cell Host Microbe, 2015. 18(3): p. 296-306.

18. Matsen, F.A., R.B. Kodner, and E.V. Armbrust, pplacer: linear time maximumlikelihood and Bayesian phylogenetic placement of sequences onto a fixed reference tree. BMC Bioinformatics, 2010. 11: p. 538.

19. Lu, J., P. Shi, and H. Li Generalized Linear Models with Linear Constraints for Microbiome Compositional Data. ArXiv e-prints, 2018.

20. Crost, E.H., et al., The mucin-degradation strategy of Ruminococcus gnavus: The importance of intramolecular trans-sialidases. Gut Microbes, 2016. 7(4): p. 302-312.

21. Berry, D.A., Bayesian clinical trials. Nat Rev Drug Discov, 2006. 5(1): p. 27-36.

22. Donald, B. and D. Rubin, Fisher, Neyman, and Bayes at FDA. Journal of Biopharmaceutical Statistics, 2016. 26: p. 1020-1024.

23. Wasserstein, R. and N. Lazar, The ASA's Statement on p-Values: Context, Process, and Purpose. The American Statistician, 2016. 70: p. 129-133.

24. Kass, R. and A. Raftery, Bayes Factors. J. Am. Stat. Assoc., 1995. 90: p. 773795.

25. Friedman, J. and B. Popescu, Predictive learning via rule ensembles. Ann. Appl. Stat., 2008. 2: p. 916-954.

26. Letham, B., et al., Interpretable classifiers using rules and Bayesian analysis: Building a better stroke prediction model. Ann. Appl. Stat., 2015. 9: p. 13501371.

27. Urbanowicz, R. and J. Moore, Learning Classifier Systems: A Complete Introduction, Review, and Roadmap. J. Artif. Evol. Appl. , 2009: p. 1-25.

28. Polson, N., J. Scott, and J. Windle, Bayesian Inference for Logistic Models Using Pólya-Gamma Latent Variables. J. Am. Stat. Assoc. , 2013. 108: p. 1339-1349.

29. Gibson, T. and G. Gerber, Robust and Scalable Models of Microbiome Dynamics, in Thirty-fifth International Conference on Machine Learning. 2018: Stockholm.

30. Callahan, B.J., et al., DADA2: High-resolution sample inference from Illumina amplicon data. Nat Methods, 2016. 13(7): p. 581-3.

31. Cole, J.R., et al., Ribosomal Database Project: data and tools for high throughput rRNA analysis. Nucleic Acids Res, 2014. 42(Database issue): p. D633-42.

32. Schloss, P.D., et al., Introducing mothur: open-source, platform-independent, community-supported software for describing and comparing microbial communities. Appl Environ Microbiol, 2009. 75(23): p. 7537-41. 
873

874

875

876

877

878

879
33. Kozich, J.J., et al., Development of a dual-index sequencing strategy and curation pipeline for analyzing amplicon sequence data on the MiSeq Illumina sequencing platform. Appl Environ Microbiol, 2013. 79(17): p. 5112-20.

34. Pedregosa, F., et al., Scikit-learn: Machine Learning in Python. J. Mach. Learn. Res., 20122. 12: p. 2825-2830.

\section{Figures}

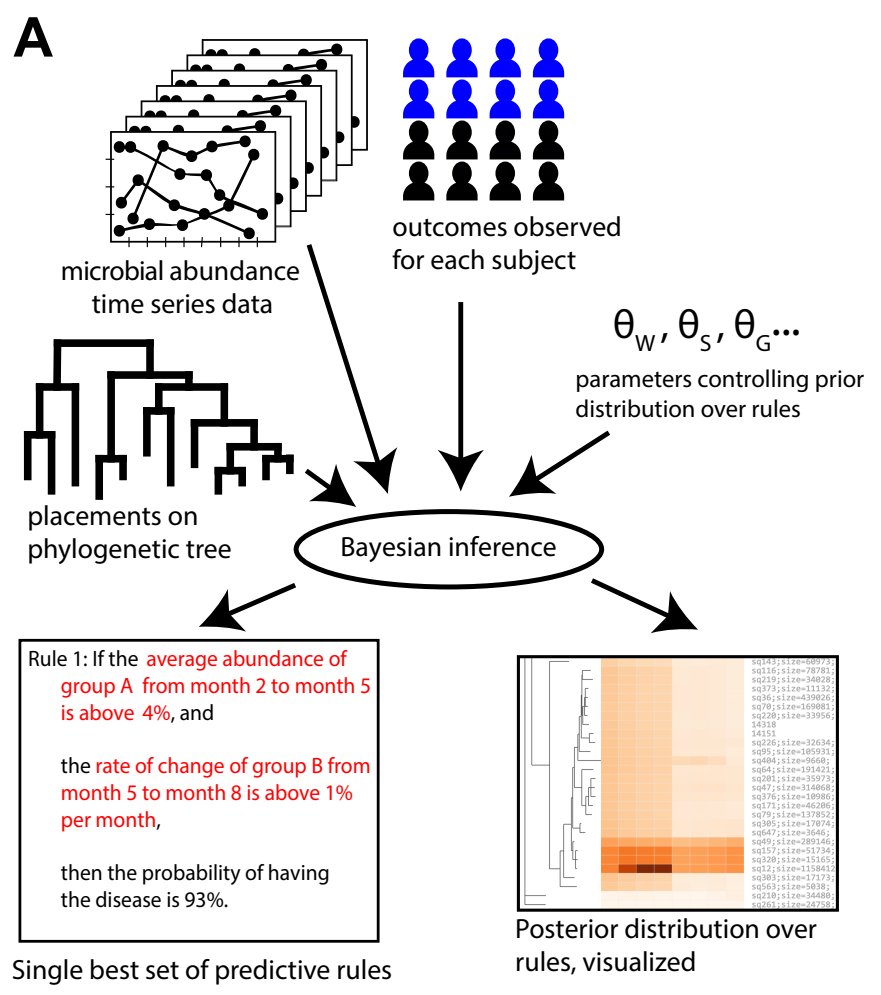

Single best set of predictive rules

Posterior distribution over
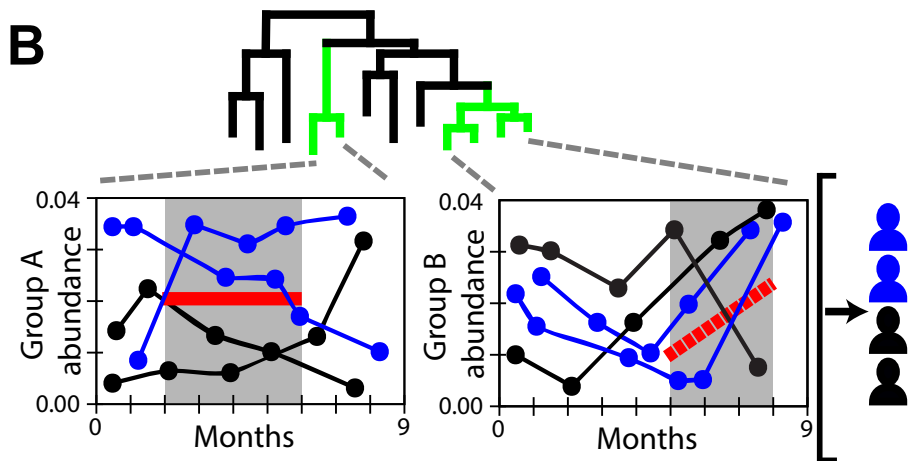

881 Figure 1: MITRE learns human-interpretable rule-based models predicting

882 host status from microbiota time-series data. Rules operate on automatically 
883 learned time-periods and groups of phylogenetically related microbes. (A) 884 Schematic of the MITRE analysis pipeline, resulting in a single best predictive 885 model as well as a distribution over alternative models that can be interactively 886 explored. (B) Schematic of example rule in (A) applied to hypothetical data. Here, 887 two subjects satisfy both the condition on the average abundance of microbe group 888 A and the rate of change of abundance of group $B$.

A

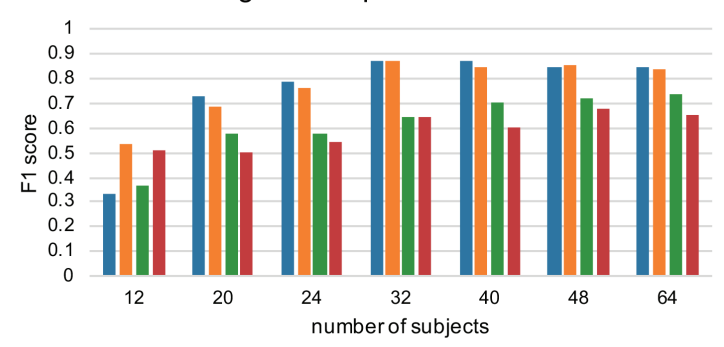

$\backsim$ MITRE ensemble $\quad$ MITRE point $\quad$-L1-reg log reg $\quad$ random forest

C

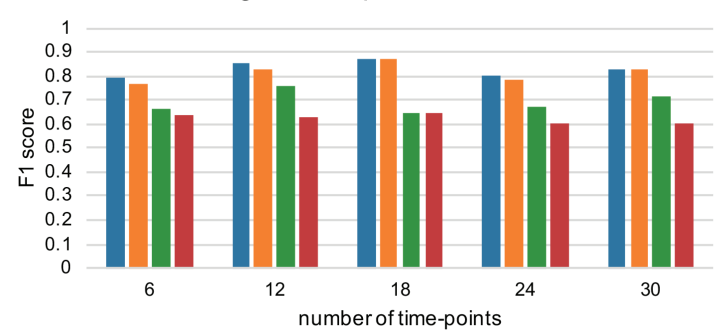

B two clade perturbation

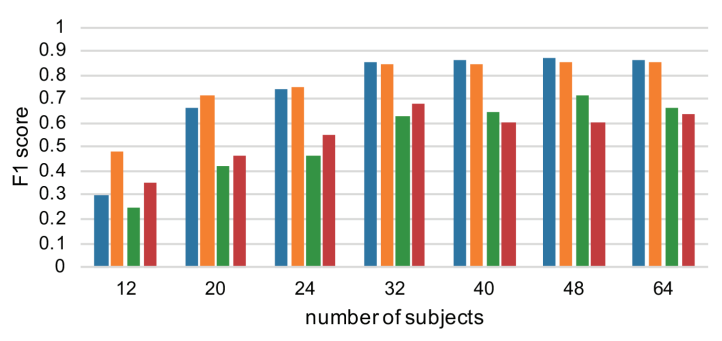

D two clade perturbation

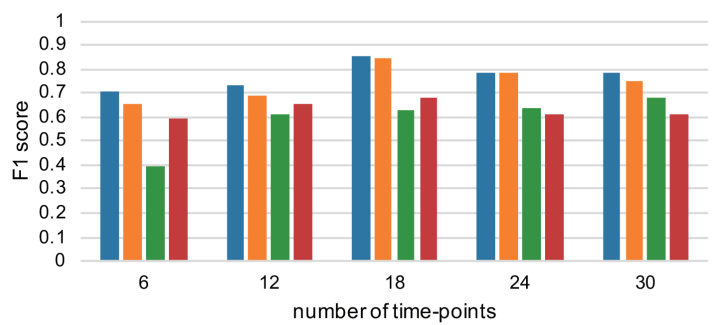

E

real data

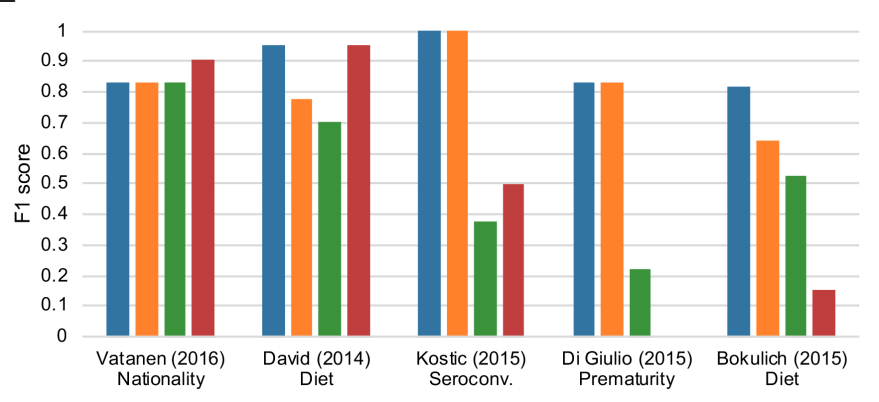


893 data. A parametric bootstrapping-type method was used to generate simulated

894 data from an underlying real dataset. Simulated cases were generated by

895 randomly selecting and perturbing bacterial clades over a randomly selected

896 limited time-window ( $20 \%$ of the duration of the study); an equal number of

897 control subjects were simulated. For the one clade perturbation scenarios, the

898 clade remained unperturbed for the simulated cases; for the two clade

899 perturbation scenarios, one clade was perturbed in the simulated control

900 subjects, and both were perturbed in the simulated cases. (A-B) One or two

901 clades randomly perturbed in simulated subjects, 18 time-points, varying

902 numbers of subjects. (C-D) One or two clades randomly perturbed in simulated

903 subjects, 32 subjects, varying numbers of time-points. (E) Results on real data.

904 The different methods were used to predict the indicated categories in the

905 datasets shown. F1-score is the harmonic mean of precision and recall; higher

906 scores indicate superior results. 


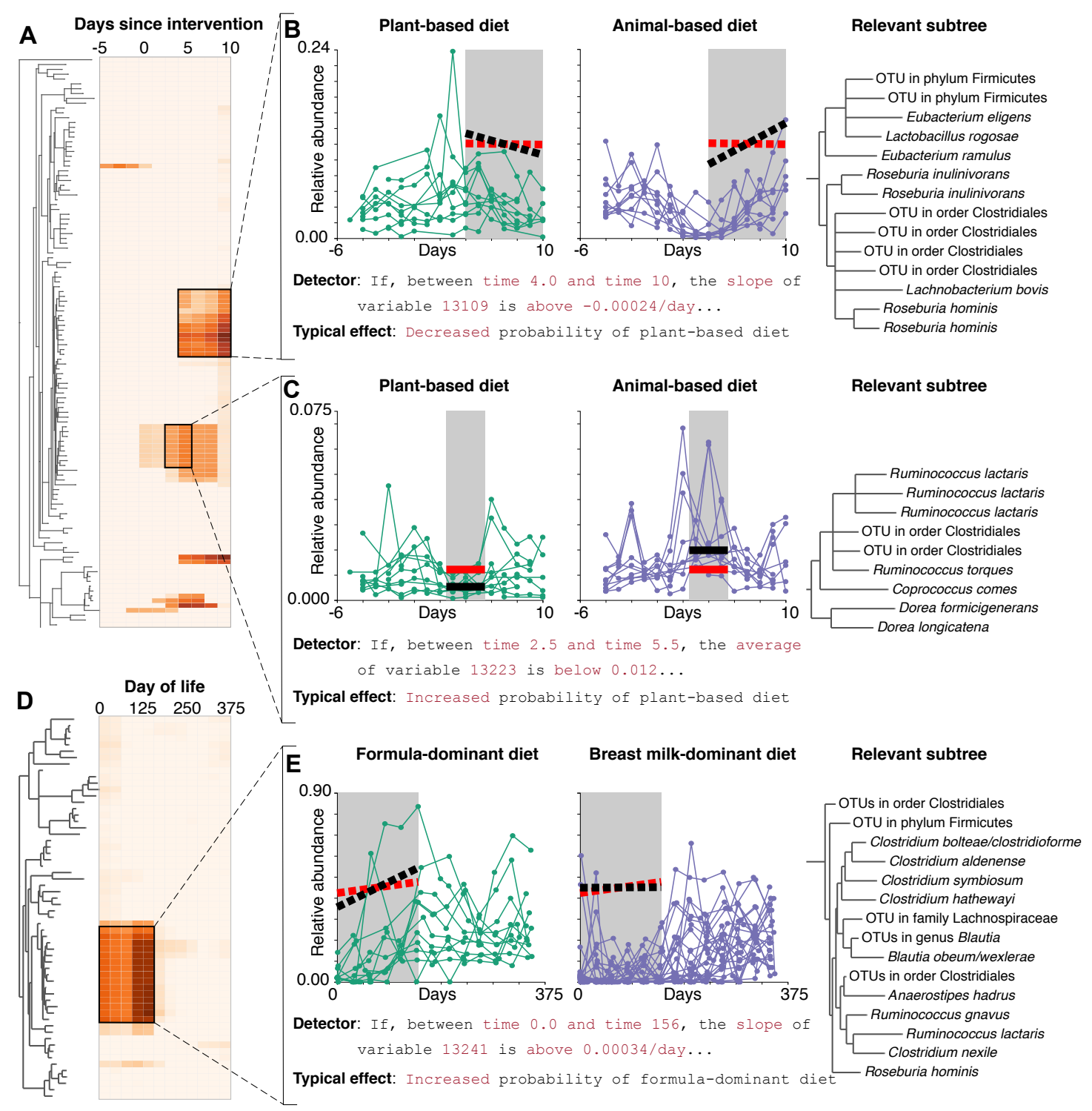

909 Figure 3. MITRE supports exploratory analyses through an interactive

910 visualization interface. The interface allows the user to explore the distribution of

911 learned rules. MITRE was applied to predict diet type from data from David et al.

912 [3] (A-C) or Bokulich et al. [2] (D-E). In (A) and (D), cell colors indicate the strength

913 of evidence that the dynamics of an OTU, or one of its ancestors, during a time

914 window is associated with diet. Panels (B), (C), and (E) show high-probability

915 detectors and phylogenetic subtrees to which they apply. (B-C) Analyses reveals 
916 dynamic behaviors of two different clades, one with butyrate producers and the 917 other without, which distinguish subjects on plant or animal-based diets. The 918 animal-based diet thus promotes two groups of phylogenetically distinct microbes 919 which are also likely functionally distinct. (E) Analyses reveal dynamic behavior of 920 a clade of bacteria, associated with a more mature microbiome, which is increased

921 in the predominantly formula-fed infants, suggesting the formula diet may shift 922 infants toward more adult-like gut microbiota. Red lines, threshold 923 slopes/abundances; black lines, median slopes/abundances. Median effect $=$ 924 median over all rules containing the detector. 